\title{
BOUNDED LINEAR OPERATORS ON BANACH FUNCTION SPACES OF VECTOR-VALUED FUNCTIONS
}

\author{
BY \\ N. E. GRETSKY AND J. J. UHL, JR.( $\left.{ }^{1}\right)$
}

\begin{abstract}
Representations of bounded linear operators on Banach function spaces of vector-valued functions to Banach spaces are given in terms of operator-valued measures. Then spaces whose duals are Banach function spaces are characterized. With this last information, reflexivity of this type of space is discussed. Finally, the structure of compact operators on these spaces is studied, and an observation is made on the approximation problem in this context.
\end{abstract}

1. Introduction and preliminaries. Over the past dozen years many vector measure representations for linear operators on spaces of integrable functions have appeared. Some have dealt with representations of linear operators on certain spaces of real-valued functions and others have dealt with representations on certain spaces of strongly measurable vector-valued functions. Often these representations have not been exploited and have stood unused in the applications. This paper has a twofold purpose. One is to unify the earlier work by obtaining a vector measure representation for the general bounded linear operator on a wide class of Banach function spaces whose members are strongly measurable functions with values in a Banach space. The second purpose is to use this representation to obtain concrete information about the function spaces involved. Specifically this paper will be concerned with characterizing those spaces under consideration whose dual is also a Banach function space of strongly measurable vector-valued functions. This information will be put to quick use in characterizing reflexive Banach function spaces of vector-valued functions. Finally, the structure of and properties of compact operators on Banach function spaces of vector-valued functions will be studied.

Throughout this paper $(\Omega, \Sigma, \mu)$ is a fixed (totally) $\sigma$-finite measure space. $M^{+}$ is the collection of all nonnegative real-valued measurable functions on $\Omega$ identified under the usual agreement that $f=g$ if $f(\omega)=g(\omega)$ for $\mu$-almost all $\omega \in \Omega$. A mapping $\rho$ on $M^{+}$to the extended real line is called a function norm if

(i) $\rho(f) \geqq 0$ and $=0$ if and only if $f=0$.

(ii) $\rho(\alpha f)=\alpha \rho(f)$ for $\alpha \geqq 0$.

Received by the editors May 10, 1971.

AMS 1970 subject classifications. Primary 46E40; Secondary 46B10, 46E30, 46G10.

Key words and phrases. Banach function spaces, vector-valued functions, representation of bounded linear operators, approximation property, Radon-Nikodym property.

(1) Supported in part under NSF GP 20431.

Copyright (C) 1972, American Mathematical Society 
(iii) $\rho(f+g) \leqq \rho(f)+\rho(g)$.

(iv) $f \leqq g$ a.e. implies $\rho(f) \leqq \rho(g)$.

$\rho$ is said to have the weak Fatou property if $f_{n} \uparrow f ; f_{n}, f \in M^{+}$; and $\sup _{n} \rho\left(f_{n}\right)<\infty$ imply $\rho(f)<\infty$. Let $\rho$ be a fixed function norm with the weak Fatou property. If $f$ is a real-valued measurable function on $\Omega$, define $\rho(f)$ by $\rho(f)=\rho(|f|)$. Let $L_{\rho}=$ $\{f \mid f: \Omega \rightarrow R, f$ measurable, $\rho(f)<\infty\}$. Then $L_{\rho}$ is a Banach space with norm $\rho$ and is called a Banach function space. All $L^{p}(1 \leqq p \leqq \infty)$ spaces and Orlicz spaces are Banach function spaces.

A set $E \subset \Omega$ is called unfriendly [9] relative to $\rho$ if for every measurable $B \subset E$ of positive $\mu$-measure, $\rho\left(\chi_{B}\right)=\infty$. There is a set $E_{0} \in \Sigma$ which is maximal with respect to the property of being unfriendly [9]. If this set is removed nothing is changed in $L_{\rho}$ since $f \in L_{\rho}$ implies $f \chi_{E_{0}}=0$ a.e. Therefore, it will be assumed that $\Sigma$ contains no unfriendly sets.

The dual norm [10] $\rho^{\prime}$ of a function norm $\rho$ is defined by

$$
\rho^{\prime}(f)=\sup \left\{\left|\int f g d \mu\right|: \rho(g) \leqq 1\right\} .
$$

The associate space $L_{\rho^{\prime}}$ is defined by $L_{\rho^{\prime}}=\left\{f: f\right.$ is measurable and $\left.\rho^{\prime}(f)<\infty\right\} . L_{\rho^{\prime}}$ is a Banach function space and there are no unfriendly sets with respect to $\rho^{\prime}$ [9]. The usual Hölder inequality holds for $L_{\rho}, L_{\rho^{\prime}}$, i.e. $f \in L_{\rho}, g \in L_{\rho^{\prime}}$ implies $\int_{\Omega}|f g| d \mu$ $\leqq \rho(f) \rho^{\prime}(g)$ [10]. $\Sigma_{0}$ is the $\delta$-ring of sets $E$ in $\Sigma$ such that both $\mu(E)$ and $\rho\left(\chi_{E}\right)$ are finite. A sequence of sets $\Delta=\left\{\Omega_{n}, n=1,2, \ldots\right\}$ in $\Sigma$ is called $\rho$-admissible if $\Omega_{n} \in \Sigma_{0}$ and $\Omega_{n} \uparrow \Omega$. The existence of such sequences is guaranteed since the measure space is $\sigma$-finite and contains no unfriendly sets with respect to $\rho$. Define $\Sigma_{00}=\Sigma_{0} \cap \Sigma_{0}^{\prime}$, i.e. $\Sigma_{00}$ is the $\delta$-ring of sets $E$ in $\Sigma$ such that $\mu(E), \rho\left(\chi_{E}\right)$, and $\rho^{\prime}\left(\chi_{E}\right)$ are finite. Absence of unfriendly sets (relative to $\rho$ and $\rho^{\prime}$ ) guarantees that $\Omega$ can be written as a countable union of sets from $\Sigma_{00}$, i.e. that there are sequences which are both $\rho$ and $\rho^{\prime}$-admissible.

DEFINITION 1.1. The space of functions of absolutely continuous norm, $L_{\rho}^{\alpha}$, is the subset of $L_{\rho}$ consisting of all $f \in L_{\rho}$ such that $\rho\left(f_{n}\right) \rightarrow 0$ for every sequence $\left\{f_{n}\right\}$ in $L_{\rho}$ such that $|f| \geqq f_{1} \geqq f_{2} \geqq \cdots \rightarrow 0$ holds pointwise a.e. on $\Omega$.

$L_{\rho}^{\alpha}$ is a closed subspace of $L_{\rho}$, but some caution is necessary since if $L_{\rho}=L^{\infty}$ and $(\Omega, \Sigma, \mu)$ is nonatomic then $L_{\rho}^{\alpha}=\{0\}$.

Definition 1.2. (i) Let $\Delta=\left\{\Omega_{n}, n=1,2, \ldots\right\}$ be a $\rho$-admissible sequence. Define $L_{\rho}^{\Delta}$ to be the subset of $L_{\rho}$ consisting of the closure of the functions in $L_{\rho}$ which are bounded and have support in some $\Omega_{n} \in \Delta$.

(ii) Define $M_{\rho}$ to be the subset of $L_{\rho}$ consisting of the closure of the functions in $L_{\rho}$ which are bounded and have support in $\Sigma_{0}$.

For a more complete discussion of the properties of $L_{\rho}^{\alpha}, L_{\rho}^{\Delta}$, and $M_{\rho}$, see Luxemburg [10], Luxemburg and Zaanen [9], and Gretsky [7]. What is needed for the present is that

$$
L_{\rho}^{\alpha}=\bigcap_{\Delta} L_{\rho}^{\Delta} \text { and } M_{\rho} \supseteq \bigcup_{\Delta} L_{\rho}^{\Delta} \text {, }
$$


where the intersection and union are over the collection of all $\rho$-admissible sequences, and equality holds in the second if (for example) either $\mu(\Omega)<\infty$ or $\mu(\Omega)=\infty, \rho\left(\chi_{\Omega}\right)=\infty$, and $\rho^{\prime}$ has property (J). (See Definition 1.3 below.) Note that $M_{\rho}$ is the closure of the $\mu$-simple functions, while $\bigcup_{\Delta} L_{\rho}^{\Delta}$ is the closure of the $\mu$-integrable $\mu$-simple functions.

Definition 1.3. A partition $\pi$ is a finite disjoint subset $\left\{E_{n}\right\}$ of $\Sigma_{00}$. If $\pi=\left\{E_{n}\right\}$ is a partition and $f \in L_{\rho}, E_{\pi}(f)=f_{\pi}$ is defined by

$$
\sum \frac{\int_{E_{n}} f d \mu}{\mu\left(E_{n}\right)} \chi_{E_{n}}
$$

where $\chi_{E_{n}}$ is the indicator of $E_{n}$ and the convention $0 / 0=0$ is employed. A function norm $\rho$ has the property $(\mathrm{J})$ if for each partition $\pi, \rho\left(f_{\pi}\right) \leqq \rho(f)$.

If $\rho$ has (J), then so does $\rho^{\prime}[7]$.

The following assumption will be in force without further mention throughout this paper.

(A) $\rho$ has (J).

This assumption is not severe. All $L^{p}$ spaces $(1 \leqq p \leqq \infty)$ and Orlicz spaces satisfy (A). For a discussion of properties of Banach function spaces see [19, Chapter 15].

Throughout this paper, $X$ is a Banach space with dual space $X^{*}$. A function $f: \Omega \rightarrow X$ is called simple if it is of the form $\sum_{i=1}^{n} x_{i} \chi_{E_{t}}$ for $x_{i} \in X, E_{i} \in \Sigma ; f$ is strongly measurable if it is the limit in $\mu$-measure of a sequence of simple functions. The integral employed here will be the Bochner integral ([6, Chapter 3] and [8]).

Definition 1.4. $L_{\rho}(X)=\{f: \Omega \rightarrow X$ is strongly measurable and $\rho(\|f\|)<\infty\}$. $L_{\rho}^{\alpha}(X)$ is the subset of $L_{\rho}(X)$ such that $f \in L_{\rho}^{\alpha}(X)$ if $\|f\| \in L_{\rho}^{\alpha} . M_{\rho}(X)$ and $L_{\rho}^{\Delta}(X)$ are defined similarly.

Under the usual identification of functions which agree except on a $\mu$-null set, $L_{\rho}^{\alpha}(X)$ becomes a Banach space and $L_{\rho}^{\alpha}(X), L_{\rho}^{\Delta}(X)$, and $M_{\rho}(X)$ are closed subspaces of $L_{\rho}(X)$. As in the scalar case, the $\mu$-simple functions are dense in $M_{\rho}(X)$, while the $\mu$-integrable $\mu$-simple functions are dense in $\bigcup_{\Delta} L_{\rho}^{\Delta} X$.

For a good exposition of the basic properties of $L_{\rho}(X)$, see Mills [12]. The $L_{\rho}(X)$ spaces furnish important examples of Banach spaces which arise as completions of the tensor product of a Banach lattice and a Banach space under an appropriate cross norm.

2. Representation theorems. This section is devoted to representation theorems for members of $B\left(\cup_{\Delta} L_{\rho}^{\Delta}(X), Y\right)$ and $B\left(L_{\rho}^{\alpha}(X), Y\right)$. The main result represents the former space as a space of $B(X, Y)$-valued set functions; specialization yields the latter space as a space of measures. Related work from a different point of view has been considered by Rao [14].

Now, let $t \in B\left(\bigcup_{\Delta} L_{\rho}^{\Delta}(X), Y\right)$. For $E \in \Sigma_{0}$, define $G(E)$ by

$$
G(E)[x]=t\left(x \chi_{E}\right), \quad x \in X .
$$


Then $G(E): X \rightarrow Y$ is obviously linear. Moreover,

$$
\|G(E)[x]\|=\left\|t\left(x \chi_{E}\right)\right\| \leqq\|t\| \rho\left(x \chi_{E}\right)=\|t\|\|x\| \rho\left(\chi_{E}\right)
$$

for $x \in X$; whence $G(E) \in B(X, Y)$. Another examination reveals that for $x \in X$, $E_{1}, E_{2} \in \Sigma_{0}, E_{1} \cap E_{2}=\varnothing$, the following equalities hold:

$$
G\left(E_{1} \cup E_{2}\right)[x]=t\left(x \chi_{E_{1} \cup E_{2}}\right)=t\left(x \chi_{E_{1}}\right)+t\left(x \chi_{E_{2}}\right)=\left(G\left(E_{1}\right)+G\left(E_{2}\right)\right)[x] .
$$

Hence $G$ is finitely additive on the $\delta$-ring $\Sigma_{0}$. Moreover, if $\mu(E)=0$ then $G(E)=0$ (the zero operator in $B(X, Y)$ ). We shall refer to this as "vanishing" on $\mu$-null sets. Thus $t \mapsto G$ sets up a correspondence between members of $B\left(\bigcup_{\Delta} L_{\rho}^{\Delta}(X), Y\right)$ and $B(X, Y)$-valued set functions which are finitely additive and "vanish" on $\mu$-null sets. Next the relationship between the action of $t$ and its corresponding operatorvalued set function $G$ will be investigated.

To this end, let $f \in \bigcup_{\Delta} L_{\rho}^{\Delta}(X)$. Then for any partition $\pi$,

$$
t\left(f_{\pi}\right)=t\left(\sum_{\pi} \frac{\int_{E_{i}} f d \mu}{\mu\left(E_{i}\right)} \chi_{E_{i}}\right)=\sum_{\pi} \frac{t\left(\left(\int_{E_{i}} f d \mu\right) \chi_{E_{i}}\right)}{\mu\left(E_{i}\right)}=\sum_{\pi} \frac{G\left(E_{i}\right)\left[\int_{E_{i}} f d \mu\right]}{\mu\left(E_{i}\right)} .
$$

Now, since $\lim _{\pi} \rho\left(f-f_{\pi}\right)=0, \lim _{\pi}\left\|t\left(f_{\pi}\right)-t(f)\right\|=0$ and

$$
t(f)=\lim _{\pi} \sum_{\pi} \frac{G\left(E_{i}\right)\left[j_{E_{i}} f d \mu\right]}{\mu\left(E_{i}\right)}
$$

is a representation of $t$ in terms of its corresponding set function $G$. This representation motivates the following question: What Banach space of vector measures is isometrically isomorphic to $B\left(L_{\rho}^{\alpha}(X), Y\right)$ under the correspondence established above? The next paragraph discusses this question.

One obvious way to sort out the required space of set functions is to define, for a $B(X, Y)$-valued set function $G$,

$$
\|G\|=\sup \left\{\left\|\sum_{i=1}^{n} G\left(E_{i}\right)\left[x_{i}\right]\right\|: x_{i} \in X, E_{i} \in \Sigma_{0}, \rho\left(\sum_{i=1}^{n} x_{i} \chi_{E_{i}}\right) \leqq 1\right\} .
$$

This is nothing more than saying that $\|G\|=\sup \{\|t(f)\|: f$ simple, $\rho(f) \leqq 1\}$ if $t \mapsto G$ as above. Some authors have chosen this norm in the $L^{\rho}(X)$ case and it does give the desired norm to the vector measures in question. On the other hand, it is just translating properties of $t$ over to the representing set function $G$. It is analogous to defining the norm in $L^{\infty}$,

$$
\|f\|_{\infty}=\sup \left\{\int_{\Omega} f g d \mu: g \in L^{1},\|g\|_{1} \leqq 1\right\} \text { for } f \in L^{\infty},
$$

rather than defining the $L^{\infty}$ norm directly as

$$
\|f\|_{\infty}=\inf \{\alpha>0: \mu(\{x:\|f\|>\alpha\})=0\} .
$$

Such a procedure skirts the issue. In addition it tends to obscure the structure of the space in question. What is needed here is a direct approach which defines an independent space of measures endowed with its own intrinsic structure and then relates this space to $B\left(\cup_{\Delta} L_{\rho}^{\Delta}(X), Y\right)$. This is the goal of the following definitions. 
Definition 2.1. Let $Z$ be a Banach space and $\rho$ be a function norm. If $G: \Sigma_{0} \rightarrow Z$ is a finitely additive set function, the $\rho$-variation of $G$ is

$$
\rho(G)=\sup _{\pi} \rho\left(\sum_{\pi} \frac{G\left(E_{i}\right)}{\mu\left(E_{i}\right)} \chi_{E_{i}}\right)
$$

where the supremum is taken over all partitions $\pi \subset \Sigma_{00}$.

Actually this is simply $\lim _{\pi} \rho\left(\sum_{\pi}\left(G\left(E_{i}\right) / \mu(E)\right) \chi_{E_{i}}\right)$ since $\rho$ has property (J). (It is easily seen that if $\rho$ has $(\mathrm{J})$, then $\rho\left(f_{\pi_{1}}\right) \leqq \rho\left(f_{\pi_{2}}\right)$ whenever $\pi_{2}$ is a finer partition than $\pi_{1}$.) The following definition furnishes the required set of measures.

Definition 2.2. Let $\rho$ be a function norm. (Recall, in this paper, $\rho$ is assumed to have the weak Fatou property and (J).) $U_{\rho^{\prime}}(B(X, Y))$ is the space of all $B(X, Y)$ valued set functions on $\Sigma_{0}$ such that

(a) $G$ "vanishes" on $\mu$-null sets,

(b) $G$ is finitely additive on $\Sigma_{0}$,

(c) $\rho^{\prime}\left(\left\|y^{*} G\right\|_{X^{*}}\right)<\infty$ for each $y^{*} \in Y^{*}$, and

(d) $\|G\|_{U_{\rho^{\prime}}}=\sup _{\left\|y^{*}\right\| \leqq 1} \rho^{\prime}\left(\left\|y^{*} G\right\|_{X^{*}}\right)<\infty$.

The above definition allows the formulation of

THEOREM 2.3. The spaces $B\left(\cup_{\Delta} L_{\rho}^{\Delta}(X), Y\right)$ and $U_{\rho^{\prime}}(B(X, Y))$ are isometrically isomorphic under the correspondence defined for $t \in B\left(\cup_{\Delta} L_{\rho}^{\Delta}(X), Y\right), G \in U_{\rho^{\prime}}(B(X, Y))$ by $t\left(x \chi_{E}\right)=G(E)[x]$ for all $x \in X, E \in \Sigma_{0}$. Moreover, if $t \leftrightarrow G$ as above then for $f \in \cup_{\Delta} L_{\rho}^{\Delta}(X)$,

$$
t(f)=\lim _{\pi} \sum_{\pi} \frac{G\left(E_{i}\right)\left[\int_{E_{i}} f d \mu\right]}{\mu\left(E_{i}\right)} .
$$

Proof. Let $t \in B\left(\bigcup_{\Delta} L_{\rho}^{\Delta}(X), Y\right)$ and let $G$ be defined as above. The early discussion of this section shows that $G$ vanishes on $\mu$-null sets and is finitely additive on $\Sigma_{0}$. Now, for any $y^{*} \in Y^{*}$ and any partition $\pi$, consider

$$
\begin{aligned}
\rho^{\prime}\left(\left\|\sum_{\pi} \frac{y^{*} G\left(E_{i}\right)}{\mu\left(E_{i}\right)} \chi_{E_{i}}\right\|_{X^{*}}\right)=\rho^{\prime}\left(\sum_{\pi} \frac{\left\|y^{*} G\left(E_{i}\right)\right\|_{X^{*}}}{\mu\left(E_{i}\right)} \chi_{E_{i}}\right) \\
=\sup \left\{\int_{\Omega}(f)\left(\sum_{\pi} \frac{\left\|y^{*} G\left(E_{i}\right)\right\|}{\mu\left(E_{i}\right)} \chi_{E_{i}}\right) d \mu: f \in L_{\rho}^{\Delta}, \rho(f) \leqq 1\right\} \\
=\sup \left\{\int_{\Omega}(f)\left(\sum_{\pi} \frac{y^{*} G\left(E_{i}\right)\left[x_{i}\right]}{\mu\left(E_{i}\right)} \chi_{E_{i}}\right) d \mu: f \in L_{\rho}^{\Delta}, \rho(f) \leqq 1,\left\|x_{i}\right\| \leqq 1\right\} \\
\leqq \sup \left\{\int_{\Omega}\left\langle f, \sum_{\pi} \frac{y^{*} G\left(E_{i}\right)}{\mu\left(E_{i}\right)}\right\rangle d \mu: f \in L_{\rho}^{\Delta}(X), \rho(f) \leqq 1\right\} \\
=\sup _{\rho(f) \leqq 1} \sum_{\pi} \frac{y^{*} G\left(E_{i}\right)\left[\int_{E_{i}} f d \mu\right]}{\mu\left(E_{i}\right)} \\
\quad\left(\operatorname{since} h^{*}\left(\int_{E_{i}} f d \mu\right)=\int_{E_{t}} h^{*}(f) d \mu \text { for all } h^{*} \in X^{*}\right) \\
=\sup _{\rho(f) \leqq 1} y^{*} t\left(f_{\pi}\right) \\
\leqq \sup _{\rho(f) \leqq 1}\left\|y^{*}\right\|\|t\| \rho\left(f_{\pi}\right) \leqq\left\|y^{*}\right\|\|t\| \quad \text { since } \rho \text { has }(\mathrm{J}) .
\end{aligned}
$$

Hence $\rho^{\prime}\left(y^{*} G\right) \leqq\|t\|\left\|y^{*}\right\|$ and $\|G\|_{U_{\rho^{\prime}}}=\sup _{\left\|y^{*}\right\| \leqq 1} \rho^{\prime}\left(y^{*} G\right) \leqq\|t\|$. 
To complete the proof of the theorem, let $G \in U_{\rho^{\prime}}(B(X, Y))$ and define $t$ on each simple function $f=\sum_{i=1}^{n} x_{i} \chi_{E}, E_{i} \in \Sigma_{0}, x_{i} \in X$ by

$$
t(f)=\sum_{i=1}^{n} G\left(E_{i}\right)\left[x_{i}\right] .
$$

Usual arguments show $t$ is well defined. Moreover, for each such simple function $f$,

$$
t(f)=\lim _{\pi} \sum_{\pi} \frac{G\left(E_{i}\right)\left[\int_{E_{i}} f d \mu\right]}{\mu\left(E_{i}\right)}
$$

trivially. Hence if $y^{*} \in Y^{*}$ and $\left\|y^{*}\right\| \leqq 1$,

$$
\begin{aligned}
y^{*} t(f) & =\lim _{\pi} \sum_{\pi} \frac{y^{*} G\left(E_{i}\right)}{\mu\left(\int_{E_{i}} f d \mu\right]}=\lim _{\pi} \int_{\Omega}\left\langle f, \sum_{\pi} \frac{y^{*} G\left(E_{i}\right)}{\mu\left(E_{i}\right)} \chi_{E_{i}}\right\rangle d \mu \\
& \leqq \rho\left(\|f\|_{X}\right) \rho^{\prime}\left(\left\|\sum_{\pi} \frac{y^{*} G\left(E_{i}\right)}{\mu\left(E_{i}\right)} \chi_{E_{i}}\right\|_{X^{*}}\right) \leqq \rho(f)\|G\|_{U_{\rho^{\prime}} \cdot}
\end{aligned}
$$

Thus $t$ is bounded on the linear manifold of $f \in \bigcup_{\Delta} L_{\rho}^{\Delta}(X)$ such that $f_{\pi}=f$ for some partition $\pi$. But for $f \in \bigcup_{\Delta} L_{\rho}^{\Delta}(X), \lim _{\pi} \rho\left(f-f_{\pi}\right)=0$. Hence, if $t$ is defined for $f \in \bigcup_{\Delta} L_{\rho}^{\Delta}(X)$ by

$$
t(f)=\lim _{\pi} t\left(f_{\pi}\right)=\lim _{\pi} \sum_{\pi} \frac{G\left(E_{i}\right)\left[\int_{E_{i}} f d \mu\right]}{\mu\left(E_{i}\right)}
$$

$t$ is extended to a member, still denoted by $t$, of $B\left(\bigcup_{\Delta} L_{\rho}^{\Delta}(X), Y\right)$, which surely satisfies

$$
t\left(x_{E}\right)=G(E)[x] \text { for } E \in \Sigma_{0}, x \in X .
$$

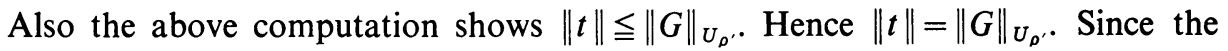
correspondence $t \leftrightarrow G$ is evidently linear, this correspondence is an isometric isomorphism between $B\left(\bigcup_{\Delta} L_{\rho}^{\Delta}(X), Y\right)$ and $U_{\rho^{\prime}}(B(X, Y))$. Q.E.D.

The representation

$$
t(f)=\lim _{\pi} \sum_{\pi} \frac{G\left(E_{i}\right)\left[\int_{E_{i}} f d \mu\right]}{\mu\left(E_{i}\right)}=\int_{\Omega} f d G
$$

is sometimes called a generalized Hellinger integral. Although in its raw form it has some utility, it does become somewhat unwieldy at times. For this reason, it is good to call attention to the fact that a small modification (no modification if $\Omega \in \Sigma_{0}$ ) of the Bartle integral [1] results in the representation

$$
t(f)=\text { Bartle- } \int_{\Omega} f d G .
$$

A somewhat more detailed theorem becomes available in the event that there is some $\Delta$ such that $L_{\rho}^{\Delta}=L_{\rho}^{\alpha}$. Luxemburg [10] showed that this is the case if and only if $L_{\rho^{\prime}} \cong\left(L_{\rho}^{\alpha}\right)^{*}$. The reason for not assuming this throughout is that common Banach function spaces such as $L^{\infty}$ or the associate spaces of the Banach function spaces discussed in [11] do not satisfy this assumption. In fact, in these three cases $L_{\rho}^{\alpha}=\{0\}$. We assume a little stronger hypothesis for the next corollary. 
Definition 2.4. Let $\rho$ be a function norm. $W_{\rho^{\prime}}(B(X, Y))$ is the space of all $B(X, Y)$-valued measures defined on $\Sigma_{0}$ such that

(a) $G$ is $\mu$-continuous,

(b) $G$ is countably additive on $\Sigma_{0}$,

(c) $\rho^{\prime}\left(y^{*} G\right)<\infty$ for each $y^{*} \in Y^{*}$,

(d) $\|G\|_{\mathrm{w}_{\rho^{\prime}}}=\sup _{\left\|y^{*}\right\| \leqq 1} \rho\left(y^{*} G\right)<\infty$.

Corollary 2.5. Assume that $L_{\rho}^{\Delta}=L_{\rho}^{\alpha}$ for every $\Delta$. Then the spaces $B\left(L_{\rho}^{\alpha}(X), Y\right)$ and $W_{\rho^{\prime}}(B(X, Y))$ are isometrically isomorphic under the correspondence defined by

$$
\begin{aligned}
G(E) & =t\left(x \chi_{E}\right), \quad x \in X, E \in \Sigma_{0}, \\
t(f) & =\lim _{\pi} \sum_{\pi} \frac{G\left(E_{i}\right)\left[\int_{E_{i}} f d \mu\right]}{\mu\left(E_{i}\right)}, \quad f \in L_{\rho}^{\alpha}(X),
\end{aligned}
$$

where $t \in B\left(L_{\rho}^{\alpha}(X), Y\right)$ and $G \in W_{\rho^{\prime}}(B(X, Y))$.

Proof. All that needs proof is that for $t \in B\left(L_{\rho}^{\alpha}(X), Y\right)$ the corresponding $G$ is countable additive and $\mu$-continuous. $G$ is countably additive in the $B(X, Y)$ topology since if $\left\{E_{n}\right\} \subset \Sigma_{0}$ and $E_{n} \downarrow \varnothing$, then

$$
\left\|G\left(E_{n}\right)\right\|=\sup _{\|x\| \leqq 1}\left\|G\left(E_{n}\right)[x]\right\| \leqq \sup _{\|x\| \leqq 1}\|t\|\|x\| \rho\left(\chi_{E_{n}}\right)
$$

implies $\lim _{n} G\left(E_{n}\right)=0$ since $\lim _{n} \rho\left(\chi_{E_{n}}\right)=0$, because $L_{\rho}^{\alpha}=\bigcup_{\Delta} L_{\rho}^{\Delta}$. The same computation also shows $\lim _{\mu(E) \rightarrow 0}\|G(E)\|=0$ on $\Sigma_{0}$. Q.E.D.

From Theorem 2.4 there is a corollary concerning $\left(\cup_{\Delta} L_{\rho}^{\Delta}(X)\right)^{*}$. This shall not concern us here. What is of interest is the following. For a Banach space $X$ and a function norm $\rho$, denote by $V_{\rho^{\prime}}(X)$ the space of all $\mu$-continuous countably additive set functions $G: \Sigma_{0} \rightarrow X$ which satisfy $\rho^{\prime}(G)<\infty$. A specialization of Corollary 2.5 to the case of $B\left(L_{\rho}^{\alpha}(X), R\right)$ yields the following information on the dual of $L_{\rho}^{\alpha}(X)$.

Corollary 2.6. Let $L_{\rho}^{\alpha}=L_{\rho}^{\Delta}$ for each $\Delta$. Then $\left(L_{\rho}^{\alpha}(X)\right)^{*}$ and $V_{\rho^{\prime}}\left(X^{*}\right)$ are isometrically isomorphic with

if and only if

$$
l \in\left(L_{\rho}^{\alpha}(X)\right)^{*} \leftrightarrow G \in V_{\rho^{\prime}}\left(X^{*}\right)
$$

$$
l(f)=\int_{\Omega} f d G, \quad f \in L_{\rho}^{\alpha}(X)
$$

Proof. Here $\left(L_{\rho}^{\alpha}(X)\right)^{*}=B\left(L_{\rho}^{\alpha}(X), R\right) \cong W_{\rho^{\prime}}(B(X, R))=W_{\rho^{\prime}}\left(X^{*}\right)$ which collapses to $V_{\rho^{\prime}}\left(X^{*}\right)$.

A more detailed investigation of the structure of $\left(L_{\rho}^{\alpha}(X)\right)^{*}$ is the aim of the next section.

3. The dual of $L_{\rho}^{\alpha}(X)$. If $X=R$, then with the assumption that $L_{\rho}^{\Delta}=L_{\rho}^{\alpha}$ for some $\Delta$, we have $L_{\rho}^{\alpha}(X)^{*}=\left(L_{\rho}^{\alpha}\right)^{*}=L_{\rho^{\prime}}=L_{\rho}\left(X^{*}\right)$. Throughout $\S 3$ we will assume that $L_{\rho}^{\alpha}=L_{\rho}^{\Delta}$ for all $\Delta$. The section is devoted to characterizing those Banach spaces $X$ for which $\left(L_{\rho}^{\alpha}(X)\right)^{*}=L_{\rho^{\prime}}\left(X^{*}\right)$. The fact that this equality is not true for all Banach spaces is a consequence of the following. 
ExAmple. Let $\Omega=[0,1]$ with Lebesgue measure $\mu$. Let $L_{\rho}=L^{2}$ and $X=l^{1}$. Then $L_{\rho}=L_{\rho^{\prime}} \cong L_{\rho}^{*}$. We note that $\rho$ is a function norm with Fatou property and property (J) and that $L_{\rho}^{\alpha}=L_{\rho}$. Consider $f=\left(f_{1}, f_{2}, \ldots, \ldots\right) \in L^{2}\left(l^{1}\right)$. Then

$$
\|f\|_{L^{2}\left(l^{1}\right)}=\left(\int_{\Omega}\left(\sum_{i=1}^{\infty}\left|f_{i}\right|\right)^{2} d \mu\right)^{1 / 2}<\infty .
$$

Let $\left\{\gamma_{n}\right\}$ be the Rademacher functions on $[0,1]$; i.e. if $s \in[0,1]$ and

$$
s=\sum_{n=1}^{\infty} a_{n}(s) / 2^{n}
$$

is the binary expansion of $s$, set $\gamma_{n}(s)=1-2 a_{n}(s)$. Now note that

$$
[0,1]=\left[\gamma_{n}=+1\right] \cup\left[\gamma_{n}=-1\right] \text { and } \mu\left[\gamma_{n}=+1\right]=\frac{1}{2} \text {. }
$$

Also recall the well-known fact that, as random variables, the $\left\{\gamma_{n}\right\}$ are stochastically independent. Define $l$ on $L^{2}\left(l^{1}\right)$ by

$$
l(f)=\int_{\Omega}\left(\sum_{n=1}^{\infty} \gamma_{n} f_{n}\right) d \mu, \quad f=\left(f_{1}, f_{2}, \ldots, f_{n}, \ldots\right) \in L^{2}\left(l^{1}\right) .
$$

Then $l$ is linear and $|l(f)| \leqq \int_{\Omega} \sum_{n=1}^{\infty}\left|\gamma_{n} f_{n}\right| d \mu \leqq \int_{\Omega} \sum_{n=1}^{\infty}\left|f_{n}\right| d \mu=\int_{\Omega}\|f\|_{l^{1}} d \mu \leqq$ $\left(\int_{\Omega}\|f\|_{l^{1}}^{2} d \mu\right)^{1 / 2}=\|f\|_{L^{2}\left(l^{1}\right)}$ by the Hölder inequality. Hence $l \in\left(L^{2}\left(l^{1}\right)\right)^{*}$. Now suppose there exists $g=\left(g_{1}, g_{2}, \ldots, g_{n}, \ldots\right) \in L^{2}\left(l^{\infty}\right)=L^{2}\left(\left(l^{1}\right)^{*}\right)$ such that

$$
l(f)=\int_{\Omega}\langle f, g\rangle d \mu=\int_{\Omega} \sum_{n=1}^{\infty} f_{n} g_{n} d \mu .
$$

It follows quickly that $g_{n}=\gamma_{n}$ a.e. [ $\mu$ ] for all $n$. Hence it may be assumed without loss of generality that $g=\left(\gamma_{1}, \gamma_{2}, \gamma_{3}, \ldots\right)$. But since $g \in L^{2}\left(l^{\infty}\right), g$ is in particular strongly measurable. Hence there exists a strongly measurable simple function $\varphi: \Omega \rightarrow l^{\infty}$ such that $\mu\left[\|\varphi-g\| \geqq \frac{1}{2}\right]<\frac{1}{4}$. Write $E=\left[\|\varphi-g\|<\frac{1}{2}\right]$ and set $E_{i}^{j}=\left[\gamma_{i}=j\right]$, $i=1,2, \ldots ; j= \pm 1$. Then $\mu(E)>\frac{3}{4}$ and $\mu\left(E_{i}^{j}\right)=\frac{1}{2}$ for all $i, j$. Consider now $E_{1}^{1}$ and $E_{1}^{-1}$. Clearly $E$ intersects both of these sets on a set of positive measure. Hence $\varphi$ takes two distinct values on $E$. Next note that by the independence of the $\gamma_{n}$ 's, $\mu\left(E_{1}^{j} \cap E_{2}^{k}\right)=\frac{1}{4}$ for $j, k= \pm 1$. Hence at least $\frac{3}{4}$ of the sets $\left\{E_{1}^{j} \cap E_{2}^{k}: j, k= \pm 1\right\}$ intersect $E$ on a set of positive measure. Therefore $\varphi$ takes on at least three distinct values on $E$. Proceeding, look at $\left\{E_{1}^{i} \cap E_{2}^{j} \cap E_{3}^{k} ; i, j, k= \pm 1\right\}$. There are $2^{3}$ of these each with measure $1 / 2^{3}$ by independence. Hence at least $\frac{3}{4}$ of them intersect $E$ on a set of positive measure. Hence $\varphi$ must take on at least $\left(\frac{3}{4}\right) 2^{3}$ distinct values on $E$. Continuing in this way shows that $\varphi$ must take at least $\left(\frac{3}{4}\right) 2^{n}$ distinct values on $E$ for any positive integer $n$. Hence $\varphi$ must take on an infinite number of distinct values. This contradicts the fact that $\varphi$ is simple and shows that such a $g$ cannot exist in $L^{2}\left(l^{\infty}\right)$. Thus it is not always true that $\left(L_{\rho}^{\alpha}(X)\right)^{*}$ can be identified with $L_{\rho^{\prime}}\left(X^{*}\right)$. The obvious question now is: When can $\left(L_{\rho}^{\alpha}(X)\right)^{*}$ be identified with $L_{\rho^{\prime}}\left(X^{*}\right)$ ?

Moving toward an answer to this question, reconsider the above example. Let $l$ be the linear functional defined above and suppose $l \leftrightarrow G \in V_{\rho^{\prime}}\left(l^{\infty}\right)$. A moment's 
reflection shows that there must be no strongly measurable $g \in L^{2}\left(l^{\infty}\right)$ such that for $E$ measurable

$$
G(E)=\text { Bochner- } \int_{E} g d \mu .
$$

For if there were such a $g$ then an easy computation shows $l(f)=\int_{\Omega}\langle f, g\rangle d \mu$ for all $f \in L^{2}\left(l^{1}\right)$. This fact motivates the recollection of the following concept.

Definition 3.1 [2]. A Banach space $X$ has the Radon-Nikodym property with respect to a finite measure $\mu$ if every $G: \Sigma \rightarrow X$ with the properties

(i) $G$ is $\mu$-continuous,

(ii) $G$ is countably additive, and

(iii) $G$ is of bounded variation

admits the representation $G(E)=$ Bochner- $\int_{E} g d \mu$ for some strongly measurable $g$ and all $E \in \Sigma$.

According to the Dunford-Pettis theorem [5] and the Phillips version of the Radon-Nikodym theorem [13], all Banach spaces which are separable duals of another Banach space or Banach spaces which are reflexive have the RadonNikodym property with respect to any finite measure $\mu$. On the other hand, it is easily seen that if $\mu$ is a purely atomic finite measure, then any Banach space has the Radon-Nikodym property with respect to $\mu$. The importance of the RadonNikodym property in this connection was first observed by Mills [12] who proved an important special case of the following theorem. This theorem constitutes the main result of this section.

THEOREM 3.2. Assume that $L_{\rho}^{\alpha}=L_{\rho}^{\Delta}$ for all $\Delta$. Then $\left(L_{\rho}^{\alpha}(X)\right)^{*}$ and $L_{\rho^{\prime}}\left(X^{*}\right)$ are isometrically isomorphic under the correspondence

defined by

$$
l \in\left(L_{\rho}^{\alpha}(X)\right)^{*} \leftrightarrow g \in L_{\rho^{\prime}}\left(X^{*}\right)
$$

$$
l(f)=\int_{\Omega}\langle f, g\rangle d \mu, \quad f \in L_{\rho}^{\alpha}(X),
$$

if and only if, for each set $E_{0} \in \Sigma_{00}, X^{*}$ has the Radon-Nikodym property with respect to the measure $\mu_{E_{0}}$ defined on $\Sigma \cap E_{0}$ by $\mu_{E_{0}}(E)=\mu\left(E_{0} \cap E\right)$.

Proof. (Sufficiency) Let $G \in V_{\rho^{\prime}}\left(X^{*}\right)$ and $E_{0} \in \Sigma_{00}$ be arbitrary. Let $\pi=\left\{E_{m}\right\}_{m=1}^{n}$ be a partition such that $E_{m} \subset E_{0}$ for each $m=1,2, \ldots, n$. Then

$$
\sum_{\pi}\left\|G\left(E_{m}\right)\right\|=\sum_{m=1}^{n} \sup _{\left\|x_{m}\right\| \geqq 1} G\left(E_{m}\right)\left[x_{m}\right]=\sum_{m=1}^{n} \sup _{\left\|x_{m}\right\| \leqq 1} l\left(x_{m} \chi_{E_{m}}\right),
$$

where $G \leftrightarrow l$ in the sense of Corollary 2.6,

$$
\begin{aligned}
& =\sup _{\left\|x_{1}\right\| \leqq 1,\left\|x_{2}\right\| \leqq 1, \ldots,\left\|x_{n}\right\| \leqq 1} \sum_{m=1}^{n} l\left(x_{m} \chi_{E_{m}}\right) \\
& \leqq\|l\| \sup _{\left\|x_{1}\right\| \leqq 1, \ldots,\left\|x_{n}\right\| \leqq 1} \rho\left(\left\|\sum_{m=1}^{n} x_{m} \chi_{E_{m}}\right\|\right), \text { since } \sum_{m=1}^{n} x_{m} \chi_{E_{m}} \in L_{\rho}^{\alpha}, \\
& \leqq\|l\| \rho\left(\chi_{E_{0}}\right) .
\end{aligned}
$$


Since $\pi$ is arbitrary, it follows that $G$ is of bounded variation on $E_{0}$. Since it was proved earlier (Corollary 2.5) that $G$ is $\mu$-continuous and countably additive inside $\Sigma_{00}$ sets, the fact that $X^{*}$ has the Radon-Nikodym property with respect to $\mu_{E_{0}}$ produces a $g$ defined on $E_{0}$ with $G(E)=\int_{E} g d \mu, E \subset E_{0}$. Defining $g$ in this way for each $E_{0} \in \Sigma_{00}$ and piecing the results together produces a strongly measurable $g$ such that $G(E)=\int_{E} g d \mu$ for $E \in \Sigma_{00}$. (The strong measurability follows from the fact that $\Omega$ can be written as a countable collection of $\Sigma_{00}$ sets.)

Next the $L_{\rho^{\prime}}\left(X^{*}\right)$ norm of $g$ will be computed. Since $\left(L_{\rho}^{\alpha}\right)^{*}=L_{\rho^{\prime}}, L_{\rho}^{\alpha}$ is norm determining for $L_{\rho^{\prime}}$. Hence

$$
\begin{aligned}
& \rho^{\prime}\left(\|g\|_{X^{*}}\right)=\sup \left\{\int_{\Omega} f\|g\|_{X^{*}} d \mu: f \in L_{\rho}^{\alpha}, \rho(f) \leqq 1\right\} \\
&=\sup \left\{\int_{\Omega}\langle f, g\rangle d \mu: f \in L_{\rho}^{\alpha}(X), \rho(\|f\|) \leqq 1\right\} \\
&=\sup \left\{\int_{\Omega}\langle f, g\rangle d \mu: f \Sigma_{00^{-}} \text {simple, } f \in L_{\rho}^{\alpha}(X), \rho(\|f\|) \leqq 1\right\} \\
&=\sup \left\{\int_{\Omega}\left\langle\sum_{i=1}^{n} x_{i} \chi_{E_{i}}, g\right\rangle d \mu:\left\{E_{i}\right\} \subset \Sigma_{00}\right. \text { disjoint, } \\
&\left.x_{i} \in X, \rho\left(\left\|\sum x_{i} \chi_{E_{i}}\right\|\right) \leqq 1\right\} \\
&=\sup \left\{\sum_{i=1}^{n} G\left(E_{i}\right)\left[x_{i}\right]: \text { same conditions as above }\right\},
\end{aligned}
$$

since Bochner integration and application of linear functionals commute,

$$
=\sup \left\{l\left(\sum_{i=1}^{n} x_{i} \chi_{E_{i}}\right): \text { same conditions as above }\right\}=\|l\|_{L_{\rho}^{\alpha}(X)^{*}}=\rho^{\prime}(G) .
$$

Hence $g \in L_{\rho^{\prime}}\left(X^{*}\right)$ and $\rho^{\prime}(\|g\|)=\rho^{\prime}(G)$. Consequently, $\rho^{\prime}(\|g\|)=\|l\|$, and $V_{\rho^{\prime}}\left(X^{*}\right)$ and $L_{\rho^{\prime}}\left(X^{*}\right)$ are isometrically isomorphic. Now since $g \in L_{\rho^{\prime}}\left(X^{*}\right)$, the Hölder inequality ensures that $\int_{\Omega}\langle f, g\rangle d \mu$ exists for $f \in L_{\rho}^{\alpha}(X)$ and defines a bounded linear functional on $L_{\rho}^{\alpha}(X)$. Clearly this functional agrees with $l$ on the simple functions in $L_{\rho}^{\alpha}(X)$, and hence $l(f)=\int_{\Omega}\langle f, g\rangle d \mu$ for all $f \in L_{\rho}^{\alpha}(X)$. This completes the proof of the sufficiency.

(Necessity) Let $E_{0} \in \Sigma_{00}$ be a fixed set of positive measure. Let $H$ be a countably additive $\mu$-continuous $X^{*}$-valued measure defined on the $\Sigma_{00}$-sets which are subsets of $E_{0}$. $\left(\Sigma_{00} \cap E_{0}\right.$.) Let $|H|$ be the variation of $H$.

Digressing for a moment, we recall that the space ca $(T, \Gamma)$ of all bounded countably additive set functions on the $\sigma$-algebra $\Gamma$ of sets in $T$ is a Banach space under the variation norm and, in fact, is a Banach lattice under "setwise" order. Moreover, to define the greatest lower bound of $\lambda$ and $\nu$ in ca $(T, \Gamma)$ we have, for $E \in \Gamma$,

$$
(\lambda \wedge \nu)(E)=\inf \{\lambda(A)+\nu(E \backslash A) \mid A \in \Gamma, A \subseteq E\}
$$


We now return to our situation and consider $|H| \wedge n \mu_{E_{0}}$ in ca $\left(E_{0}, \Sigma_{00} \cap E_{0}\right)$ for each positive integer $n$. In this case we have that both $|H|$ and $\mu_{E_{0}}$ are nonnegative and that $|H|$ is absolutely continuous with respect to $\mu_{E_{0}}$. Let $E_{n}=$ $\left\{\omega \in E_{0}:\left(d|H| / d \mu_{E_{0}}\right)(\omega)<n\right\}$. Then, for each $n$, we have the decompositions

$$
\left(|H| \wedge n \mu_{E_{0}}\right)(E)=|H|\left(E \cap E_{n}\right)+n \mu\left(E \cap E_{n}^{c}\right) \quad \text { for } E \in \Sigma_{00} \cap E_{0} .
$$

This can be seen by noting that

$$
\begin{aligned}
\left(|H| \wedge n \mu_{E_{0}}\right)(E) & =\inf \left\{|H|(A)+n \mu_{E_{0}}(E \backslash A) \mid A \subseteq E\right\} \\
& =\inf \left\{\int_{A} \frac{d|H|}{d \mu_{E_{0}}} d \mu_{E_{0}}+n \int_{E \backslash A} d \mu_{E_{0}} \mid A \subseteq E\right\} \\
& =\inf \left\{\int_{E_{0}}\left(\frac{d|H|}{d \mu_{E_{0}}} \chi_{A}+n \chi_{E \backslash A}\right) \cdot d \mu_{E_{0}} \mid A \subseteq E\right\} .
\end{aligned}
$$

Since the integrand is nonnegative, it is easily seen that the integral is minimized for $A=E_{n} \cap E$, which result gives the required decomposition.

Now consider for each $n, H_{n}(E)=H\left(E \cap E_{n}\right)$. Then $H_{n}$ is a vector measure satisfying $\left\|H_{n}(E)\right\| \leqq n \mu(E)$ for all $E \in \Sigma_{00}$. It follows quickly that $H_{n} \in V_{\rho^{\prime}}\left(X^{*}\right)$. But the assumption that $L_{\rho^{\prime}}\left(X^{*}\right) \cong V_{\rho^{\prime}}\left(X^{*}\right)$ establishes $h_{n} \in L_{\rho^{\prime}}\left(X^{*}\right)$ such that

$$
H_{n}(E)=\int_{E} h_{n} d \mu \text { for } E \in \Sigma_{00} \cap E_{0}
$$

Clearly each $h_{n}$ vanishes outside $E_{n}$ and $h_{n} \chi_{E_{m}}=h_{m}$ a.e. for $n \geqq m$. Now define $h$ on $E_{0}$ by

$$
\begin{aligned}
h(\omega) & =h_{1}(\omega) & & \text { if } \omega \in E_{1}, \\
& =h_{n+1}(\omega) & & \text { if } \omega \in E_{n+1}-E_{n}, n>1, \\
& =0 & & \text { otherwise. }
\end{aligned}
$$

Then $h$ is strongly measurable. Moreover, since $H$ is $\mu$-continuous, $\mu\left(E_{0}-\bigcup_{n} E_{n}\right)$ $=0$. Hence the monotone convergence theorem implies

$$
\int_{E_{0}}\|h\|_{X^{*}} d \mu=\lim _{n} \int_{E_{n}}\|h\| d \mu=\lim _{n}|H|\left(E_{n}\right)=|H|\left(E_{0}\right) .
$$

Hence $h$ is Bochner integrable. Finally, if $E \in \Sigma_{00} \cap E_{0}$,

$$
\begin{aligned}
\int_{E} h d \mu & =\lim _{n} \int_{E \cap E_{n}} h d \mu=\lim _{n} \int_{E \cap E_{n}} h_{n} d \mu \\
& =\lim _{n} H_{n}\left(E \cap E_{n}\right)=\lim _{n} H\left(E \cap E_{n}\right)=H(E) .
\end{aligned}
$$

This proves the necessity. Q.E.D.

In view of the discussion preceding the statement of Theorem 3.2 the following corollaries are immediate:

COROLlaRY 3.3. Assume $L_{\rho}^{\alpha}=L_{\rho}^{\Delta}$ for each admissible sequence $\Delta$. If $X$ is reflexive or if $X^{*}$ is separable, or if $\mu$ is purely atomic, then $\left(L_{\rho}^{\alpha}(X)\right) \cong L_{\rho^{\prime}}\left(X^{*}\right)$. 
The following corollary has been obtained by Mills [12] with a separability condition imposed on $X$.

Corollary 3.4. $L_{\rho}(X)$ is reflexive if and only if both $L_{\rho}$ and $X$ are reflexive.

4. Compact members of $B\left(L_{\rho}^{\alpha}(X), Y\right)$. This section is concerned with applications of the earlier representation theorems to the problem of characterizing the compact members of $B\left(L_{\rho}^{\alpha}(X), Y\right)$. The work here bears some similarity to the work of [17] where the related problem for Orlicz spaces is treated. Also included in this section are some comments on the approximation problem and the existence of a projection of $B\left(L_{\rho}^{\alpha}(X) Y\right)$ onto the space of compact members of $B\left(L_{\rho}^{\alpha}(X), Y\right)$.

THEOREM 4.1. Suppose that the $\mu$-integrable, simple functions are dense in $L_{\rho^{\prime}}$ and suppose $\left(L_{\rho}^{\alpha}(X)\right)^{*} \cong L_{\rho^{\prime}}\left(X^{*}\right)$. Then $t \in B\left(L_{\rho}^{\alpha}(X), Y\right)$ is compact if and only if

(i) for each $E_{0} \in \Sigma_{0}$ the operator $T(E): X \rightarrow Y$ defined by $T(E)[x]=t\left(x \chi_{E}\right)$ is compact, and

(ii) $\lim _{\pi}\left\|t \circ E_{\pi}-t\right\|=0$ in the uniform operator topology.

Note that the hypothesis on $L_{\rho^{\prime}}$ will be satisfied if $L_{\rho^{\prime}}=L_{\rho^{\prime}}^{\alpha}$ or if $L_{\rho^{\prime}}=\bigcup_{\Delta} L_{\rho^{\prime}}^{\Delta}$.

Proof. (Sufficiency) Let $\pi=\left\{E_{i}\right\}$ be a partition. Then for $f \in L_{\rho}^{\alpha}(X)$,

$$
t \cdot E_{\pi}(f)=t\left(\sum_{\pi} \frac{\int_{E_{i}} f d \mu}{\mu\left(E_{i}\right)} \chi_{E_{i}}\right)=\sum_{\pi} \frac{t\left(\int_{E_{i}} f d \mu \chi_{E_{i}}\right)}{\mu\left(E_{i}\right)}=\sum_{\pi} \frac{T\left(E_{i}\right)\left[\int_{E_{i}} f d \mu\right]}{\mu\left(E_{i}\right)} .
$$

By (i), $T\left(E_{i}\right)$ sends bounded sets into conditionally compact sets in $Y$. Now if $\rho(\|f\|) \leqq 1, f \in L_{\rho}^{\alpha}(X)$, then $\left\|\int_{E_{t}} f d \mu\right\| \leqq \rho^{\prime}\left(\chi_{E_{i}}\right)$ by the Hölder inequality. Hence

$$
\left\{\int_{E_{\mathfrak{i}}} f d \mu: \rho(f) \leqq 1\right\}
$$

is a bounded set in $X$ for each $E_{i}$. Thus $\left\{T\left(E_{i}\right)\left[\int_{E_{i}} f d \mu\right]: \rho(\|f\|) \leqq 1, f \in L_{\rho}^{\alpha}(X)\right\}$ is conditionally compact in $Y$ for each $E_{i}$. Looking at the form of $t \cdot E_{\pi}$ above, one sees immediately that $t \cdot E_{\pi}$ is compact. By (ii), $\lim _{\pi}\left\|t \cdot E_{\pi}-t\right\|=0$. Hence $t$ is compact.

(Necessity) Suppose $t \in B\left(L_{\rho}^{\alpha}(X), Y\right)$ is compact. For $E \in \Sigma_{0}$, consider $S=$ $\{T(E)[x]:\|x\| \leqq 1\}$. Since $T(E)[x]=t\left(x \chi_{E}\right)$ for $x \in X$ and since $\left\{x \chi_{E}:\|x\| \leqq 1\right\}$ is a bounded set in $L_{\rho}^{\alpha}(X)$, the compactness of $t$ ensures the conditional compactness of $S$. This proves the necessity of condition (i).

To establish (ii), let $y^{*} \in Y^{*}$ be fixed. Then $y^{*} t \in\left(L_{\rho}^{\alpha}(X)\right)^{*} \cong L_{\rho^{\prime}}\left(X^{*}\right)$ under the current hypothesis. Hence there exists a $g \in L_{\rho^{\prime}}\left(X^{*}\right)$ such that, for all $f \in L_{\rho}^{\alpha}(X)$,

$$
y^{*} t(f)=\int_{\Omega}\langle f, g\rangle d \mu .
$$

Now for the same $y^{*}$, consider for a partition $\pi$,

$$
y^{*} t \cdot E_{\pi}(f)=\int_{\Omega}\left\langle E_{\pi} f, g\right\rangle d \mu=\int_{\Omega}\left\langle f, E_{\pi} g\right\rangle d \mu
$$


by a short computation. Hence if $y^{*} t \leftrightarrow g$, then $y^{*} t E_{\pi} \leftrightarrow E_{\pi} g$. But since the integrable simple functions are dense and since $\rho^{\prime}$ has $(\mathrm{J}), \lim _{\pi} E_{\pi} g=g$ strongly in $L_{\rho^{\prime}}\left(X^{*}\right)$. Thus, $y^{*} t E_{\pi} \rightarrow y^{*} t$ in $\left(L_{\rho}^{\alpha}(X)\right)^{*}$; i.e. $\left(t E_{\pi}\right)^{*} y^{*} \rightarrow t^{*} y^{*}$ in $\left(L_{\rho}^{\alpha}(X)\right)^{*}$. Therefore $\lim _{\pi}\left(t \cdot E_{\pi}\right)^{*}=t^{*}$ in the strong operator topology from $Y^{*}$ to $\left(L_{\rho}^{\alpha}(X)\right)^{*}$. Moreover, the fact that $\rho^{\prime}$ has $(\mathrm{J})$ guarantees that $\left\|\left(t \cdot E_{\pi}\right)^{*}\left(y^{*}\right)\right\|=\left\|y^{*} t \cdot E_{\pi}\right\|=$ $\rho^{\prime}\left(\left\|E_{\pi} g\right\|_{X^{*}}\right) \leqq \rho^{\prime}(\|g\|)=\left\|y^{*} t\right\|=\left\|t^{*}\left(y^{*}\right)\right\|$. Also the compactness of $t$ implies $t^{*}$ is compact. Hence by the dominated convergence theorem for compact operators [17, Theorem 4, p. 212] and Vala [18], $\lim _{\pi}\left\|\left(t \cdot E_{\pi}\right)^{*}-t *\right\|=0$. Thus

$$
\lim _{\pi}\left\|t \cdot E_{\pi}-t\right\|=\lim _{\pi}\left\|\left(t \cdot E_{\pi}\right)^{*}-t *\right\|=0
$$

as required. Q.E.D.

Theorem 4.1 has an immediate application to the problem of approximating compact operators by operators with finite rank.

COROLlARY 4.2. Under the hypothesis of Theorem 4.1 and with the additional assumption that every compact member of $B(X, Y)$ can be approximated in the uniform operator topology by members of $B(X, Y)$ with a finite dimensional range, then the same statement is true for compact members of $B\left(L_{\rho}^{\alpha}(X), Y\right)$.

Proof. Let $t \in B\left(L_{\rho}^{\alpha}(X), Y\right)$ be compact and let $\varepsilon>0$ be given. According to Theorem 4.1, there is a partition $\pi$ such that $\left\|t-t \cdot E_{\pi}\right\|<\varepsilon / 2$. Now for each $E_{n} \in \pi$, consider $T\left(E_{n}\right)[\cdot]=t\left(\cdot \chi_{E_{n}}\right) \in B(X, Y)$. By Theorem 4.1, $T\left(E_{n}\right)$ is compact. For each $n$, choose an operator of finite rank $S_{n} \in B(X, Y)$ such that $\left\|T\left(E_{n}\right) / \mu\left(E_{n}\right)-S_{n}\right\|$ $<\varepsilon / 2^{n+1}\left(\rho^{\prime}\left(\chi_{E_{n}}\right)+1\right)$; define $s$ on $L_{\rho}^{\alpha}(X)$ by $s(f)=\sum_{\pi} S_{n}\left(\int_{E_{n}} f d \mu\right)$. Then

$$
\begin{aligned}
\left\|t_{\pi}-s\right\| & =\sup _{\rho(\|f\|) \leqq 1}\left\|\left(t_{\pi}-s\right)(f)\right\| \\
& =\sup _{\rho(\|f\|) \leqq 1}\left\|\sum_{\pi}\left(\frac{T\left(E_{n}\right)}{\mu\left(E_{n}\right)}-S_{n}\right)\left[\int_{E_{n}} f d \mu\right]\right\| \\
& \leqq \sup _{\rho(\|f\|) \leqq 1} \sum_{\pi}\left\|\frac{T\left(E_{n}\right)}{\mu\left(E_{n}\right)}-S_{n}\right\| \rho(\|f\|) \rho^{\prime}\left(\chi_{E_{n}}\right),
\end{aligned}
$$

by the triangle inequality and the Hölder inequality,

$$
<\sum_{\pi}\left(\varepsilon / 2^{n+1}\right) \leqq \varepsilon / 2 .
$$

Hence $\|t-s\|<\varepsilon$, as required. Q.E.D.

The next result couples Theorem 4.1 and the representation Theorem 2.5 to show that members of $W_{\rho^{\prime}}(B(X, Y))$ corresponding to compact members of $B\left(L_{\rho}^{\alpha}(X), Y\right)$ can be identified intrinsically in $W_{\rho^{\prime}}(B(X, Y))$.

THEOREM 4.3. Let $\rho$ be a function norm such that the $\mu$-integrable simple functions are dense in $L_{\rho^{\prime}}$ and suppose $\left(L_{\rho}^{\alpha}(X)\right)^{*} \cong L_{\rho^{\prime}}\left(X^{*}\right)$. Then $G \in W_{\rho^{\prime}}(B(X, Y))$ represents a compact member of $B\left(L_{\rho}^{\alpha}(X), Y\right)$ if and only if

(i) $G$ is compact-valued, i.e. $G(E) \in B(X, Y)$ is compact for all $E \in \Sigma_{0}$, and 
(ii) if $G_{\pi}$ is defined on $\Sigma_{00}$ for each partition $\pi$ by

$$
G_{\pi}(E)=\sum_{\pi} \frac{G\left(E_{n}\right)}{\mu\left(E_{n}\right)} \mu\left(E_{n} \cap E\right),
$$

then $\lim _{\pi}\left\|G-G_{\pi}\right\|_{\mathrm{W}_{\rho^{\prime}}}=0$.

Proof. The proof follows immediately from the definition of $G$ and the fact that $G_{\pi}$ represents $t \cdot E_{\pi}$ if $G$ represents $t \in B\left(L_{\rho}^{\alpha}(X), Y\right)$.

The considerations of this section combine to give an easy proof of the following theorem.

THEOREM 4.4. Let $\rho$ be a function norm such that the $\mu$-integrable simple functions are dense in $L_{\rho^{\prime}}$. Then there is no bounded projection of $B\left(L_{\rho}^{\alpha}, Y\right)$ onto the space of all compact members of $B\left(L_{\rho}^{\alpha}, Y\right)$ unless all the members of $B\left(L_{\rho}^{\alpha}, Y\right)$ are compact.

Proof. Suppose there is a bounded projection $P$ mapping $B\left(L_{\rho}^{\alpha}, Y\right)$ onto the closed subspace of compact members of $B\left(L_{\rho}^{\alpha}, Y\right)$. Let $t \in B\left(L_{\rho}^{\alpha}, Y\right)$ be arbitrary. The argument used in the proof of Theorem 4.1 shows that $t \cdot E_{\pi}$ is compact for all partitions $\pi$. Hence $(P t) \cdot E_{\pi}=P\left(t \cdot E_{\pi}\right)=t \cdot E_{\pi}$ for all partitions $\pi$. Furthermore the hypothesis of Theorem 4.1 is obviously satisfied by the current hypothesis. According to Theorem $1, \lim _{\pi}(P t) E_{\pi}=P t$ in the uniform operator topology. Hence $\lim _{\pi} t \cdot E_{\pi}=P t$ in the uniform operator topology. But, if $f \in L_{\rho}^{\alpha}$ is arbitrary,

$$
\lim _{\pi} t \cdot E_{\pi}(f)=t\left(\lim _{\pi} E_{\pi}(f)\right)=t(f) .
$$

Hence $P t(f)=t(f)$ for all $f \in L_{\rho}^{\alpha}$, i.e. $P t=t$ for all $t \in B\left(L_{\rho}^{\alpha}, Y\right)$. Q.E.D.

This result seems to improve on some results of Tong [15] who treats the case of sequence spaces.

\section{REFERENCES}

1. R. G. Bartle, A general bilinear vector integral, Studia Math. 15 (1956), 337-352. MR 18, 289.

2. S. D. Chatterji, Martingale convergence and the Radon-Nikodym theorem in Banach spaces, Math. Scand. 22 (1968), 21-41. MR 39 \#7645.

3. M. M. Day, Normed linear spaces, 2nd rev. ed., Academic Press, New York; SpringerVerlag, Berlin, 1962. MR 26 \#2847.

4. N. Dinculeanu, Vector measures, Internat. Series on Monographs in Pure and Appl. Math., vol. 95, Pergamon Press, Oxford; VEB Deutscher Verlag, Berlin, 1967. MR 34 \#6011b.

5. N. Dunford and B. J. Pettis, Linear operations on summable functions, Trans. Amer. Math. Soc. 47 (1940), 323-392. MR 1, 338.

6. N. Dunford and J. T. Schwartz, Linear operators. I: General theory, Pure and Appl. Math., vol. 7, Interscience, New York, 1958. MR 22 \#8302.

7. N. E. Gretsky, Representation theorems on Banach function spaces, Mem. Amer. Math. Soc. No. 84 (1968).

8. E. Hille and R. S. Phillips, Functional analysis and semi-groups, rev. ed., Amer. Math. Soc. Colloq. Publ., vol. 31, Amer. Math. Soc., Providence, R. I., 1957. MR 19, 664. 
9. W. A. J. Luxemburg and A. C. Zaanen, Some remarks on Banach function spaces, Nederl. Akad. Wetensch. Proc. Ser. A 59=Indag. Math. 18 (1956), 110-119. MR 17, 987.

10. W. A. J. Luxemburg, Banach function spaces, Thesis, Technische Hogeschool te Delft, 1955. MR 17, 285.

11. W. A. J. Luxemburg and A. C. Zaanen, Some examples of normed Köthe spaces, Math. Ann. 162 (1965/66), 337-350. MR 33 \#540.

12. S. E. Mills, Banach function spaces of vector-valued functions, M.S. Thesis, Louisiana State University, Baton Rouge, La., 1970.

13. R. S. Phillips, On weakly compact subsets of a Banach space, Amer. J. Math. 65 (1943), 108-136. MR 4, 218.

14. M. M. Rao, Linear operations, tensor products and contractive projections in function spaces (preprint).

15. A. Tong, Projecting the space of bounded operators onto the space of compact operators, Proc. Amer. Math. Soc. 24 (1970), 362-365.

16. J. J. Uhl, Jr., Orlicz spaces of finitely additive set functions, Studia Math. 29 (1967), 19-58. MR 37 \#1985.

17. - Compact operators on Orlicz spaces, Rend. Sem. Mat. Univ. Padova 42 (1969), 209-219. MR 40 \#7838.

18. K. Vala, On compact sets of compact operators, Ann. Acad. Sci. Fenn. Ser. A.I. No. 351 (1964). MR 29 \#6333.

19. A. C. Zaanen, Integration, rev. ed., North-Holland, Amsterdam; Interscience, New York, 1967. MR 36 \#5286.

Department of Mathematics, University of California, Riverside, California 92507

Department of MAThematics, University of Illinois, URbana, Illinois 61801 